\title{
Are good subjective organizers good paired-associate learners?
}

\author{
DAVID S. GORFEIN \\ New College, Sarasota, Fla. 33578
}

It has been demonstrated that Ss high in subjective organization in multitrial free recall are better paired-associate learners than $S$ s low on the subjective organization variable. The results of that study were interpreted as supporting the idea that the advantage in free recall of the good organizer is the ability to form associations between verbal items more rapidly than the poor organizer. The present study employs the partial correlation technique to explore the relationship between subjective organization, paired-associated learning, and free-recall acquisition. Our analysis indicates that the interpretation of the relationship between subjective organization and paired-associate learning previously offered is invalid and based on a confounding of effects.

In a recent report, Earhard \& Endicott (1969) separated Ss into a high- and a low-performance group on a free-recall subjective organization (SO) measure. They then showed that the high-SO group performed significantly better than the low group on paired-associate tasks.

One problem with investigations of individual differences in this type of design is that, when the groups are separated on the basis of one variable, they tend to be separated on any variable highly correlated with the criterion dimension. In this case, it is known that SO correlates highly with free-recall performance. The study reported below investigates the possible confounding produced by this variable.

\section{SUBJECTS}

Thirty-two paid New College students served as $\mathrm{Ss}$.

\section{FREE RECALL 1}

A 40-item unrelated word list was presented for seven trials, with items randomly arranged on each trial. A 1 -sec rate was employed with visual presentation. Four minutes were allowed for written free recall. Each S was assigned an SO score, using Tulving's (personal communication) bidirectional subjective organization measure, and a total recall score (performance summed over all seven trials).

\section{PAIRED ASSOCIATES ${ }^{2}$}

All Ss were tested on a 15-item paired-associate list, using the numbers 1 to 15 as stimuli and medium $\mathrm{m}^{\prime}$ CVCs (Noble, 1961) as responses. Four random orders of this list were used. Ss were given standard paired-associate instructions and required to learn the list to the criterion of one perfect trial at a 2:2 rate. The PA measure was number of trials to criterion, hence a high score represents a slow learner.

\section{RESULTS}

The Ss were divided into high and low (above and below median) scorers on both the SO and total recall measures. Of the $32 \mathrm{Ss}, 28$ fell on the same side of the median for both measures. Median trials to criterion in the PA task for the high SO Ss was 12.5 and 20.5 for the low Ss. A median test indicates a significant difference in rate of learning paired associates (chi square $=6.27, \mathrm{p}<.02$ ). With the division on the basis of performance, the median trials to criterion for the high performers was 11.5 and for the low performers, 21. A median test shows a significant difference (chi square $=10.16$, $\mathrm{p}<.01$ ).

If the abilities underlying $\mathrm{SO}$ and total recall are different, it is very possible that only one of these variables is related to the ability factor determining success in PA inaning. Dividing the $S$ s at the median very often makes it impossible to separate the effects of two variables as highly related as $\mathrm{SO}$ and total recall. In cases like this, it would seem preferable to use a partial correlational technique. We'd be primarily interested in the effect of SO on PA learning rate, with differences in total scores partialed out and on the relation between PA performance and total recall with SO partialed out. The partials are: SO and PA, $.17(\mathrm{p}>.10)$, and total recall and PA, $-.45(\mathrm{p}<.02)$.

$$
\text { DISCUSSION }
$$

The analysis of our data indicates that, had we adopted the technique reported by Earhard and Endicott for separating their groups, our data would have resulted in the same conclusions. However, with the more appropriate partial correlation technique, it is clear that the significant relationship that exists in our data is between the learning of paired-associates and the performance in the free-recall situation. Subjective organization with free-recall performance partialed out fails to correlate significantly with paired-associate learning. The outcome of this analysis is particularly unfortunate because it leaves us lacking a theoretical explanation for the correlation between PA and free-recall performance.

\section{REFERENCES}

EARHARD M \& ENDICOTT, $O$. Why are there individual differences in subjective organization during free-recall memorization? Journal of Verbal Learning \& Verbal Behavior, 1969, 8, 316-319.

NOBLE, C. E. Measurement of association value (a), rated associations $\left(a^{\prime}\right)$, and scaled meaningfulness $\left(\mathrm{m}^{\prime}\right)$ for the 2100 CVC combinations of the English alphabet. Psychological Reports, 1961.8, 487-521.

\section{NOTES}

1. The free-recall data was collected as part of a senior thesis by Claudia Blair.

2. The paired-associate data was collected as part of a required independent study project by Christopher Arbak and Diana Graves. 